\title{
Amine- and Acid-Free Synthesis of Stable CsPbBr Perovskite Nanocrystals
}

Yixuan Liu, Dan Li, Lulu Zhang, Yujuan Chen, Chong Geng, ${ }^{*}$ Shuangshuang Shi, Zizhen Zhang, Wengang $\mathrm{Bi}$, and $\mathrm{Shu} \mathrm{Xu}^{*}$

Tianjin Key Laboratory of Electronic Materials and Devices, School of Electronics \& Information Engineering, Hebei University of Technology, 5340 Xiping Road, Beichen District, Tianjin, 300401, China 

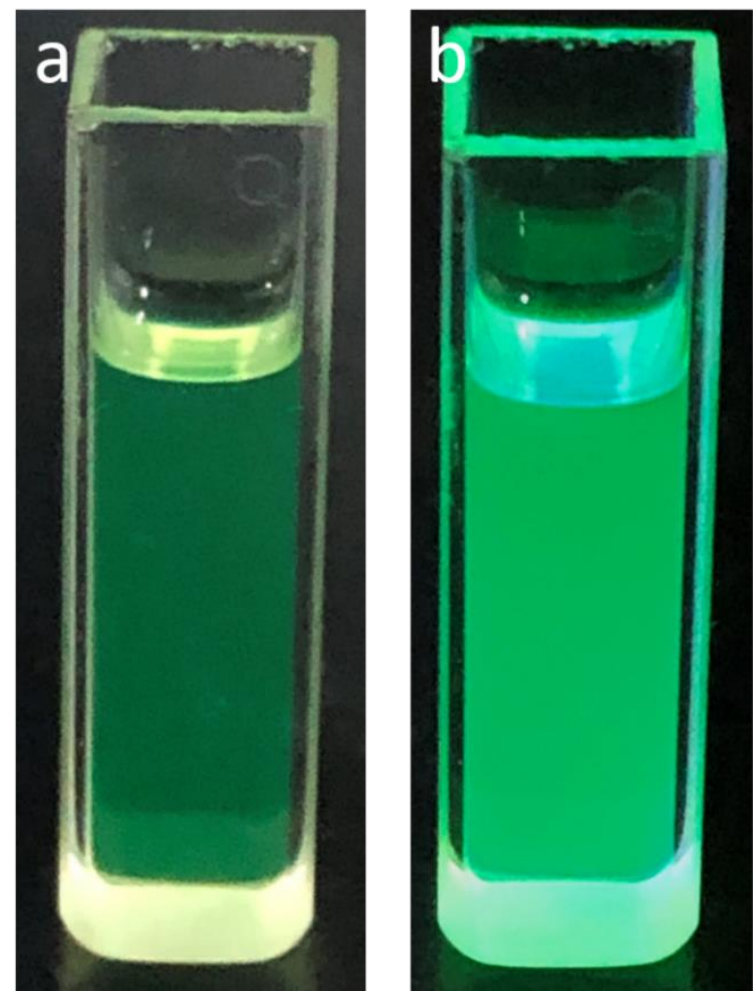

Figure S1. Photographs of I-IPNCs solutions a) under room light and b) UV light illumination.

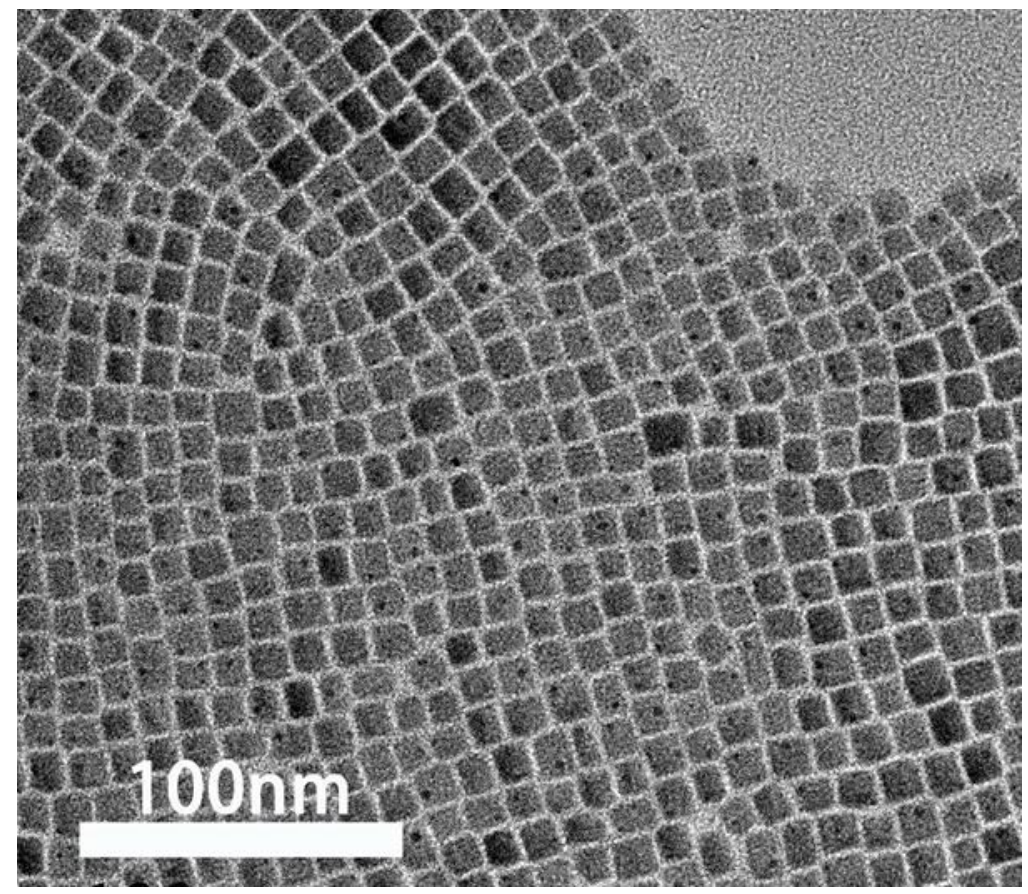

Figure S2. TEM image of I-IPNCs. 


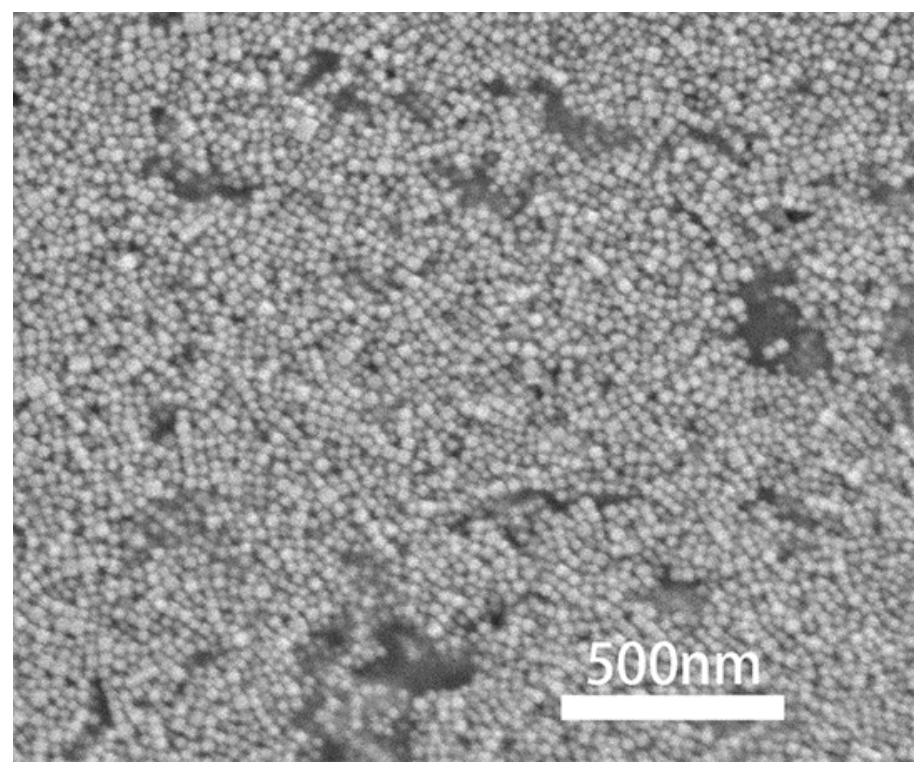

Figure S3. SEM image of C-IPNCs.

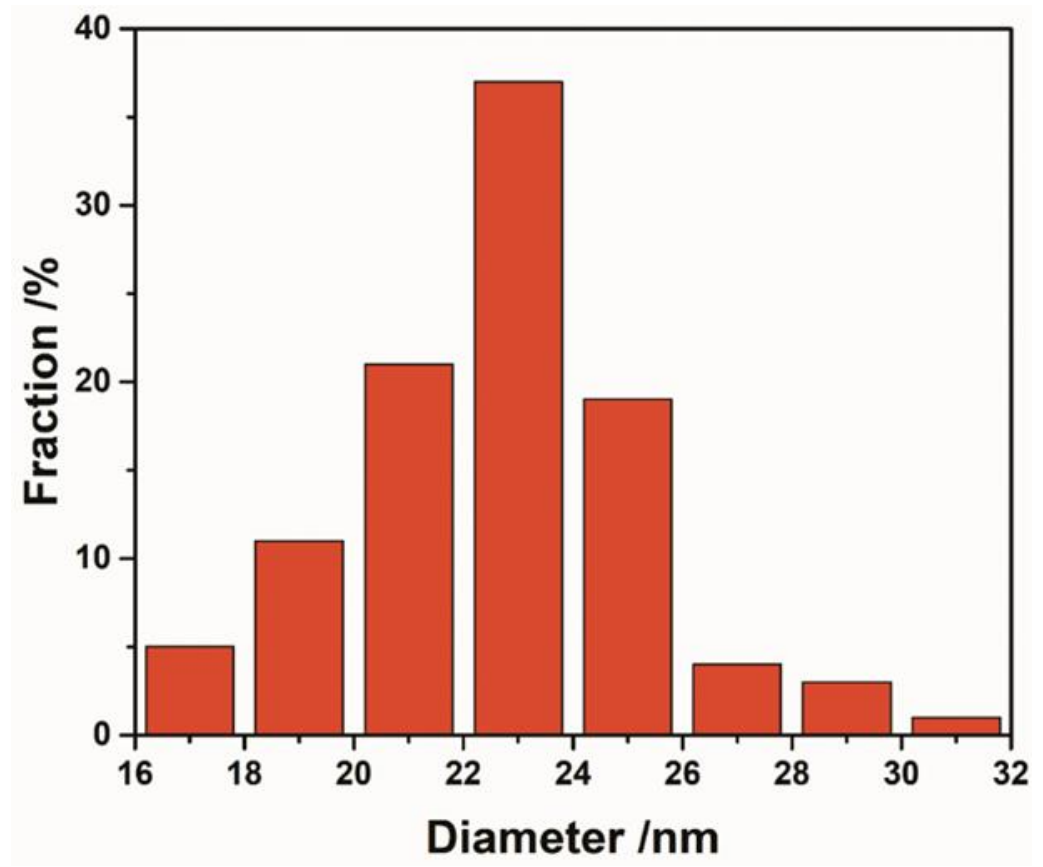

Figure S4. Size distribution of C-IPNCs obtained from the TEM characterization. 


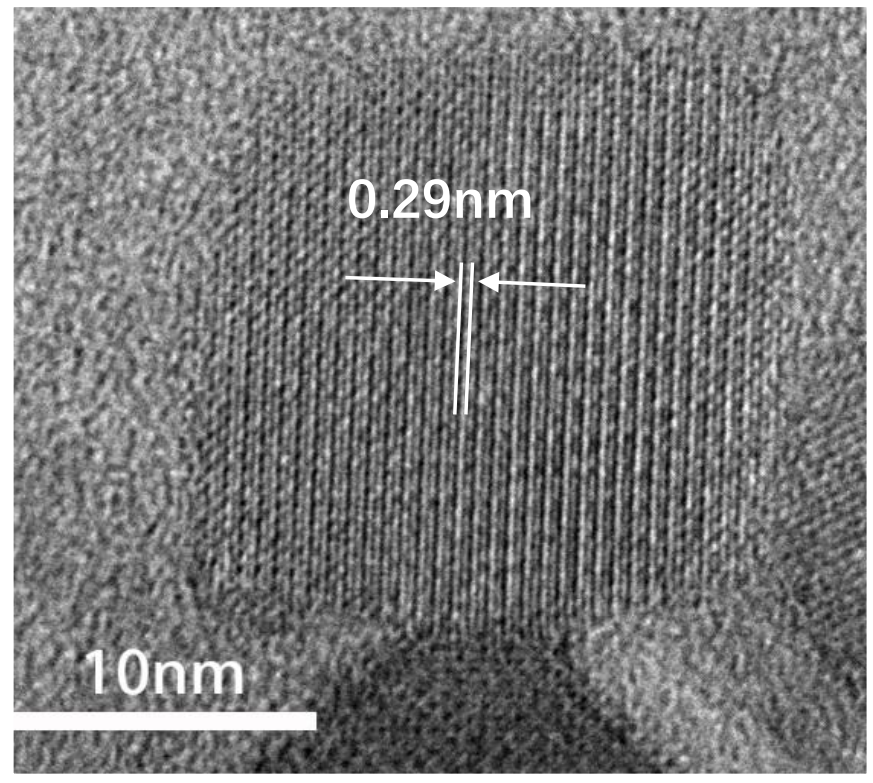

Figure S5. HRTEM image of a single C-IPNC.

Table S1. Detailed information of PL-decay of I-IPNCs and C-IPNCs.

\begin{tabular}{cccc}
\hline Sample & $\begin{array}{c}\tau_{1} \\
{[\mathrm{~ns}]}\end{array}$ & $\begin{array}{c}\tau_{2} \\
{[\mathrm{~ns}]}\end{array}$ & $\begin{array}{c}\tau_{\text {ave }} \\
{[\mathrm{ns}]}\end{array}$ \\
\hline I-IPNCs & 3.5 & 15.7 & 12.8 \\
C-IPNCs & 3.9 & 33.6 & 32.0 \\
\hline
\end{tabular}

Time-resolved PL decay curves can be fitted as biexponential decay curves:

$$
F(t)=\sum a_{i} e^{-\frac{-t}{\tau_{i}}}
$$

where $\mathrm{F}(\mathrm{t})$ is the PL intensity at time $\mathrm{t} . \mathrm{a}_{\mathrm{i}}$ and $\tau_{\mathrm{i}}$ represent the relative amplitude and the excited state lifetime of each exponential component of PL decay. The extracted excited-state lifetime, average lifetimes of each exponential component of the PL decay are summarized in Table S1. The lifetime parameters $\tau_{1}$ represents the intrinsic exciton recombination of cubic phase $\mathrm{CsPbBr}_{3}$ IPNCs $^{1}$ and $\tau_{2}$ is the results of the surface trap state mediate process. ${ }^{2}$ 

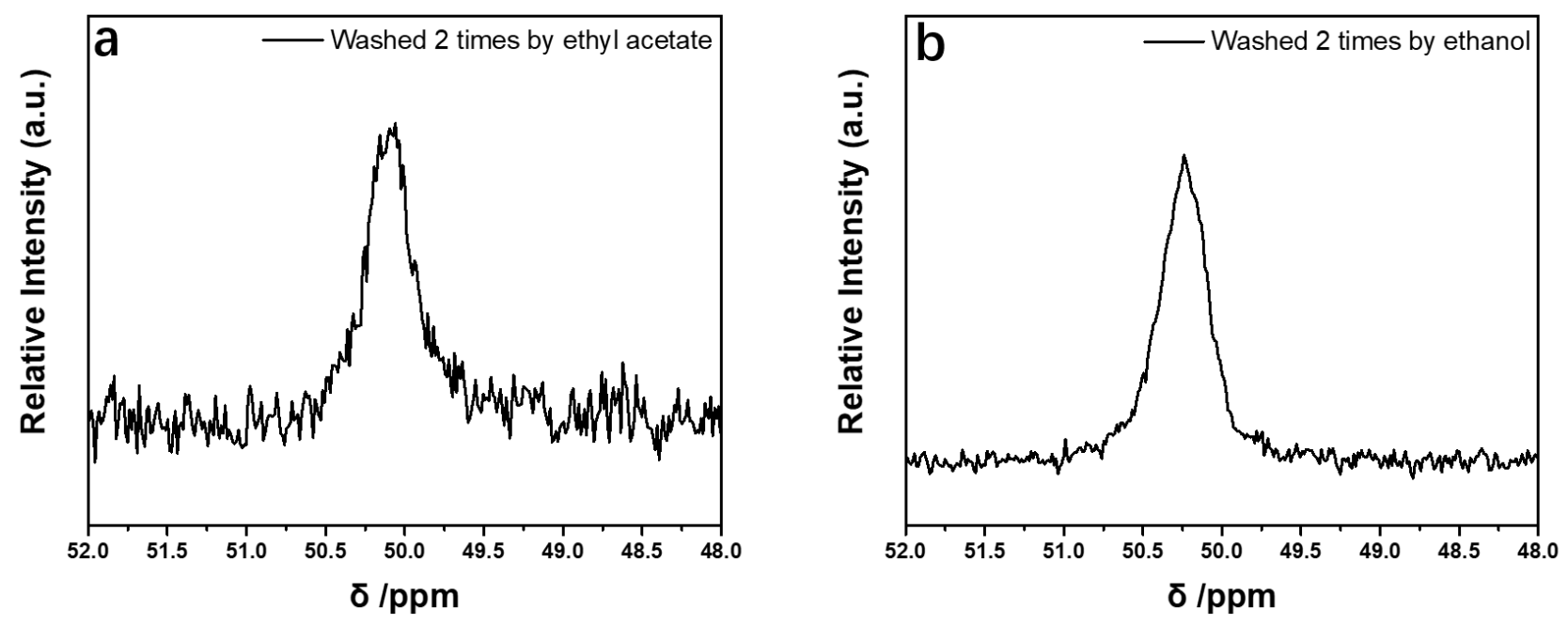

Figure S6. ${ }^{31}$ P NMR spectra of C-IPNCs after washed twice by a) ethyl acetate and b) ethanol. For NMR measurements, the samples were washed, precipitated and redispersed in deuterated chloroform inside the glovebox, then the tube is sealed for the test. The solution ${ }^{31} \mathrm{P}$ NMR test is performed at room temperature at $295 \mathrm{~K}$ operating at $400 \mathrm{MHz}$.

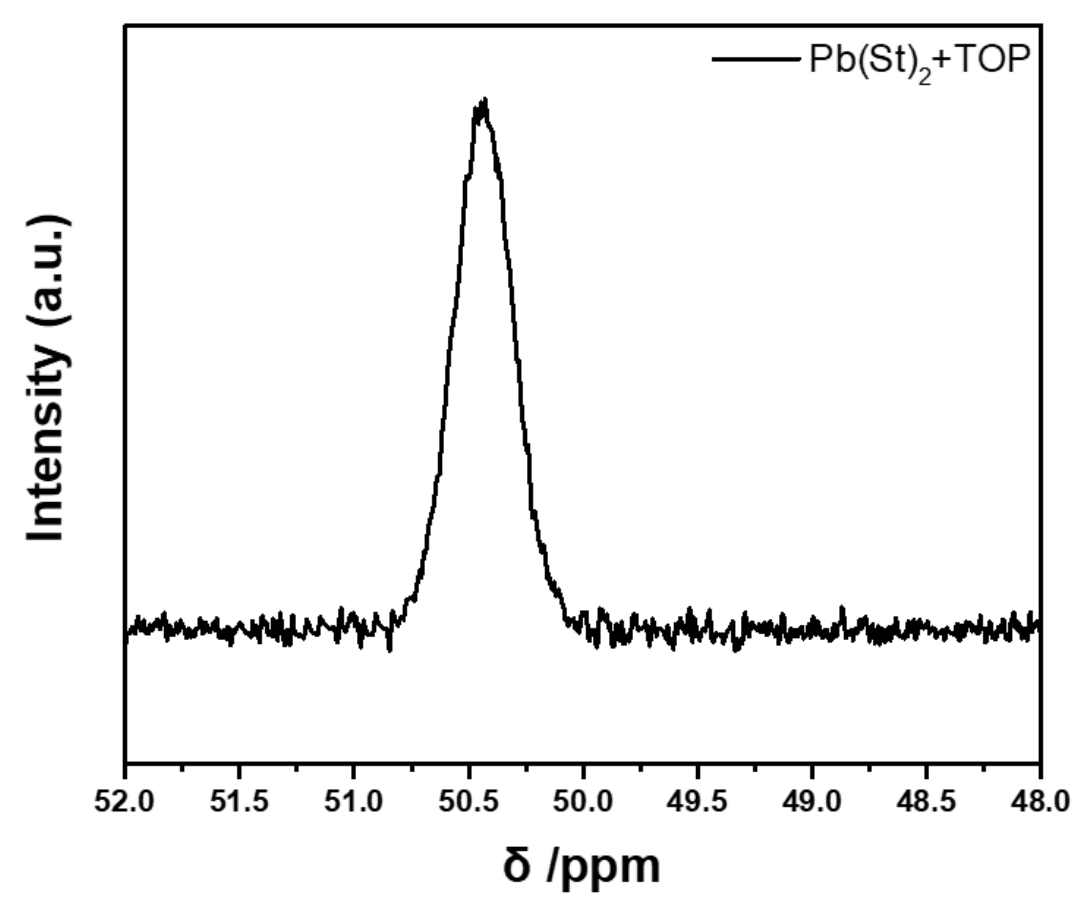

Figure S7. ${ }^{31} \mathrm{P}$ NMR of the product of $\mathrm{TOP}+\mathrm{Pb}(\mathrm{St})_{2}$. 


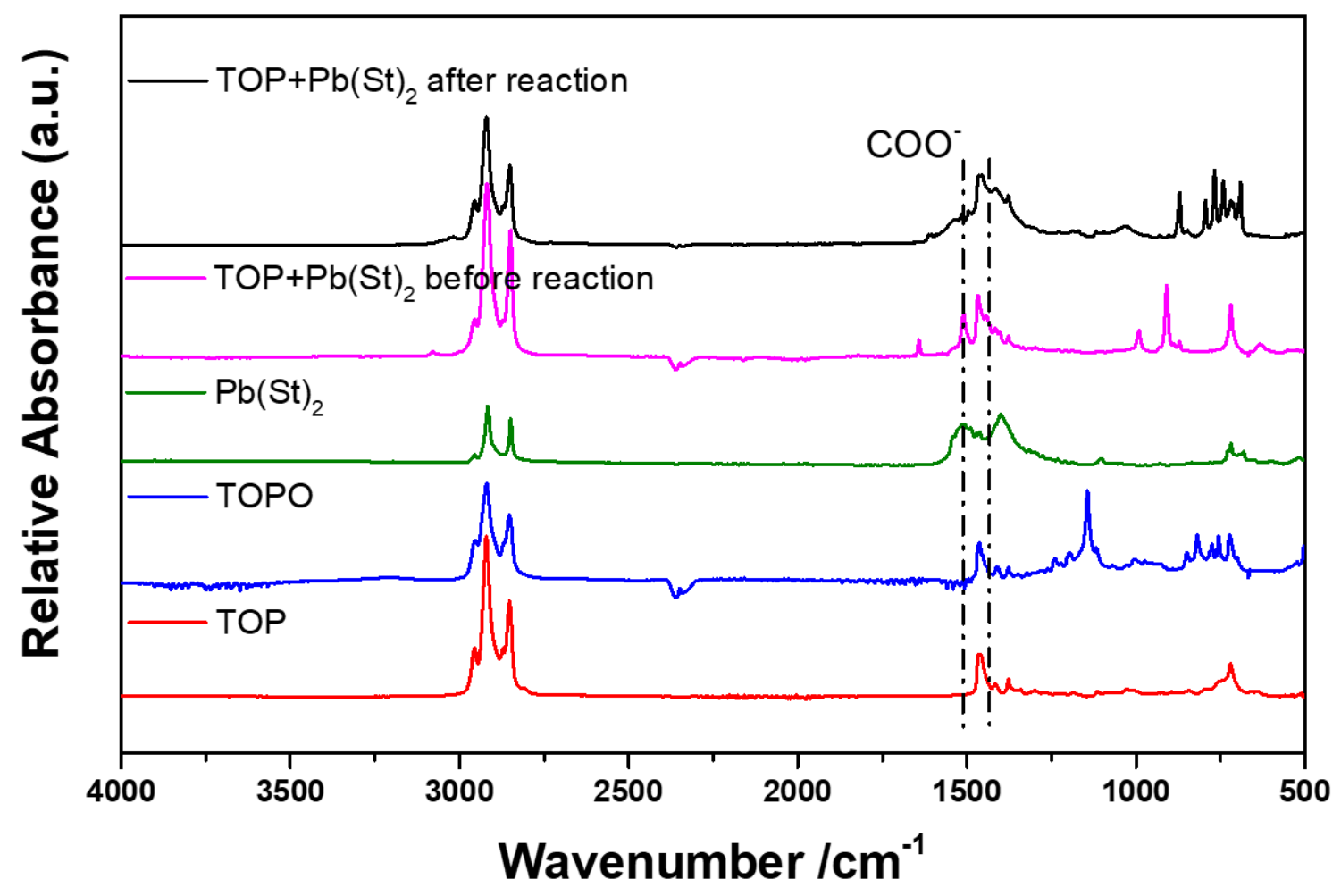

Figure S8. IR spectra of TOP, $\mathrm{TOPO}, \mathrm{Pb}(\mathrm{St})_{2}, \mathrm{TOP}+\mathrm{Pb}(\mathrm{St})_{2}$ before and after the reaction.
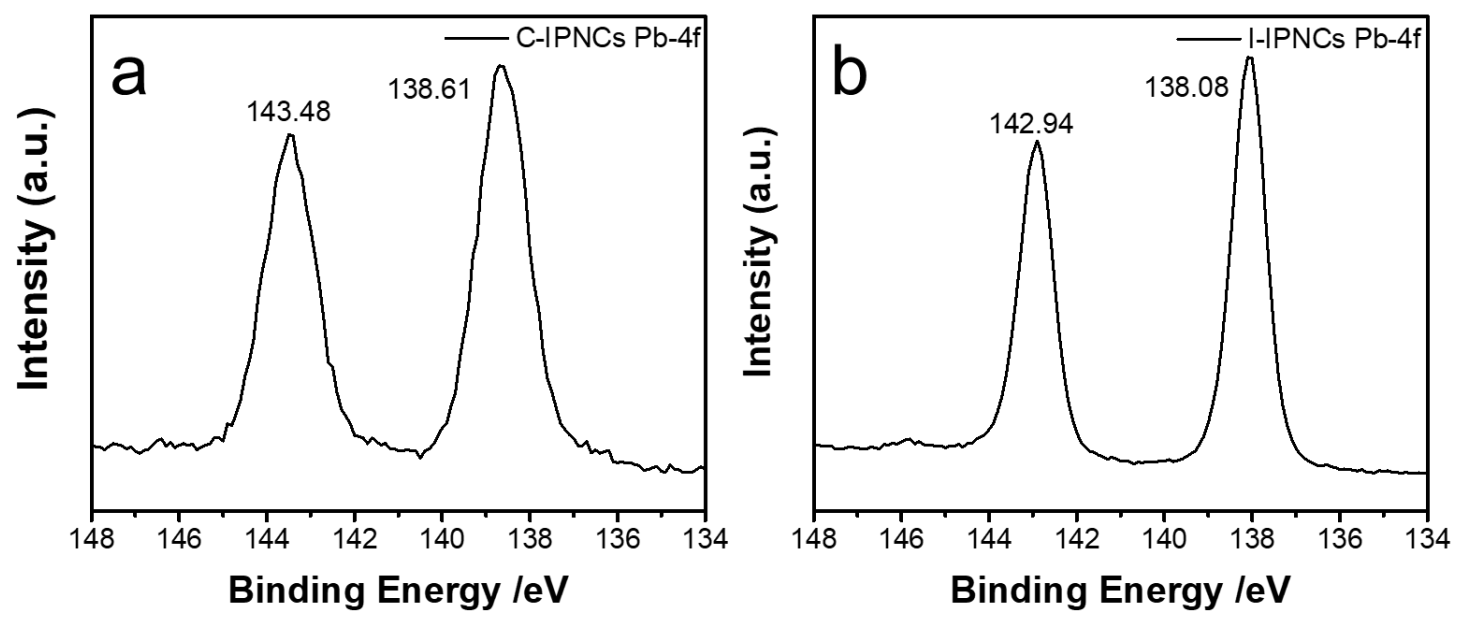

Figure S9. High-resolution XPS of Pb-4f chemical state of a) C-IPNCs and b) I-IPNCs. 

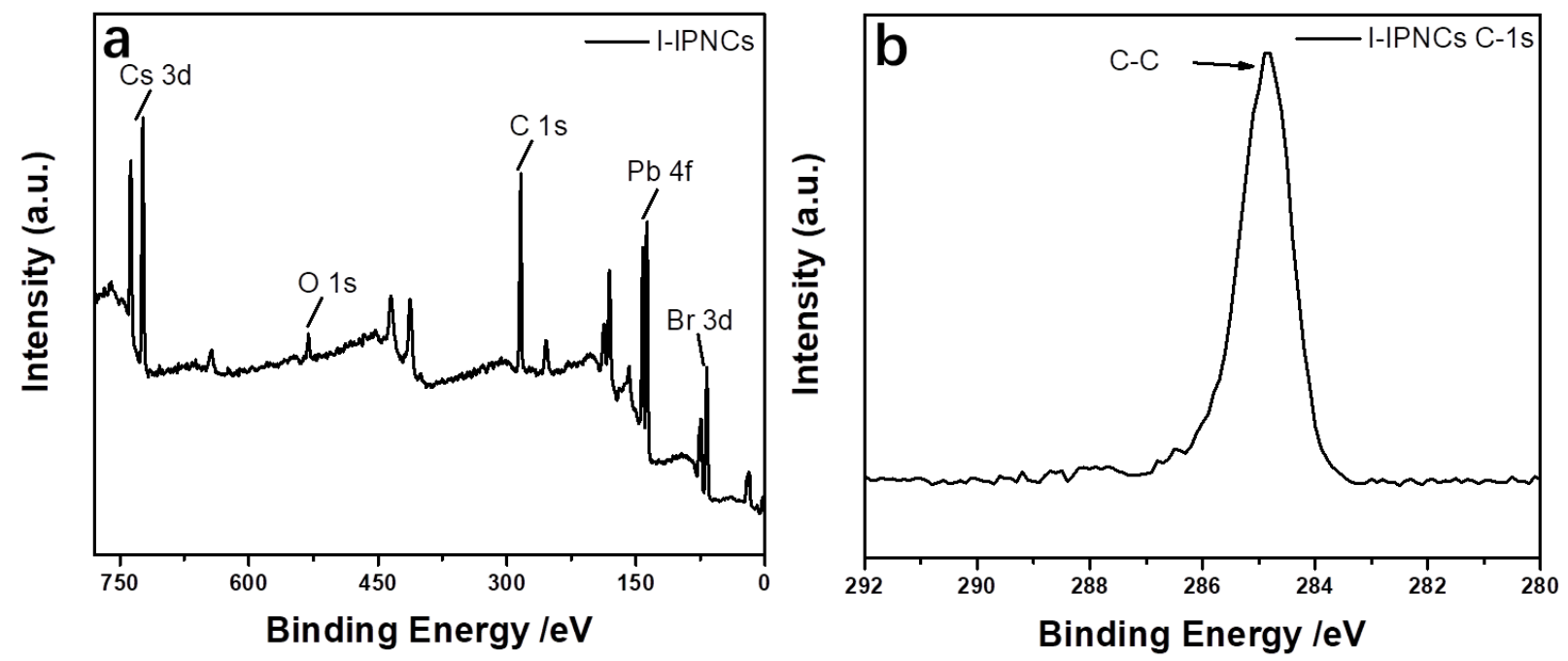

Figure S10. a) XPS survey spectrum and b) high-resolution XPS of C 1s chemical state of I-IPNCs.
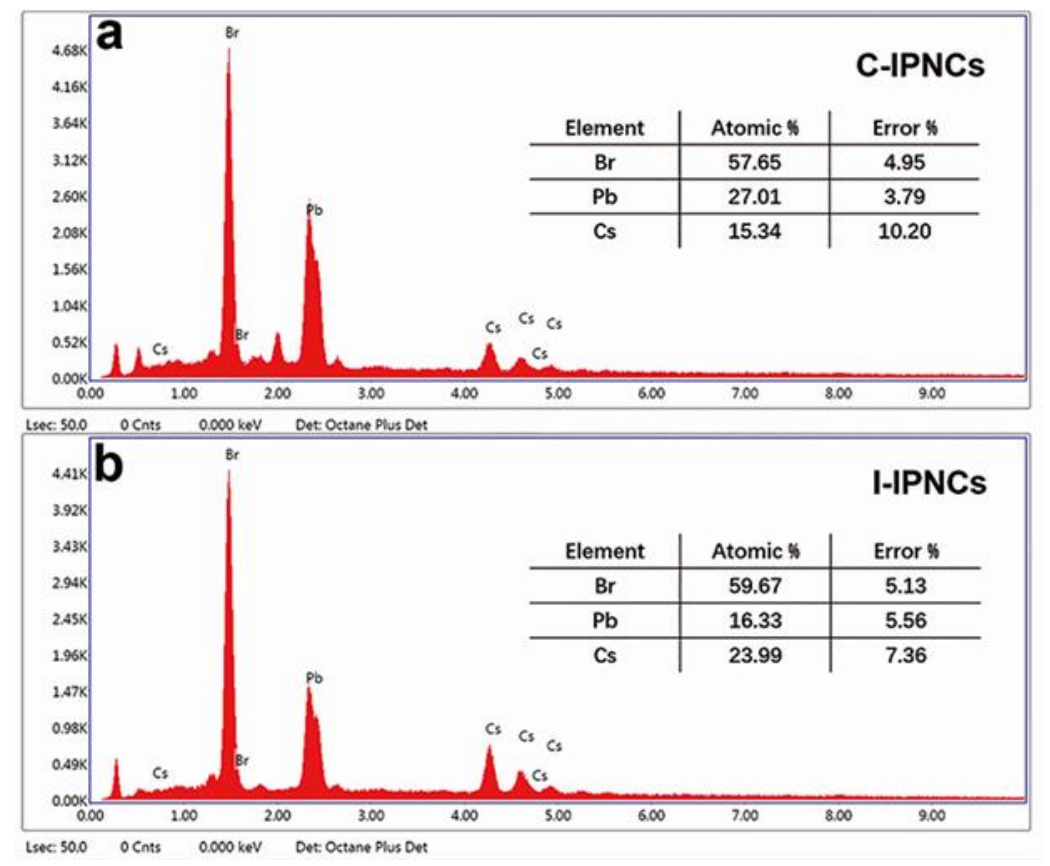

Figure S11. EDS characterization results of C-IPNCs and I-IPNCs. 

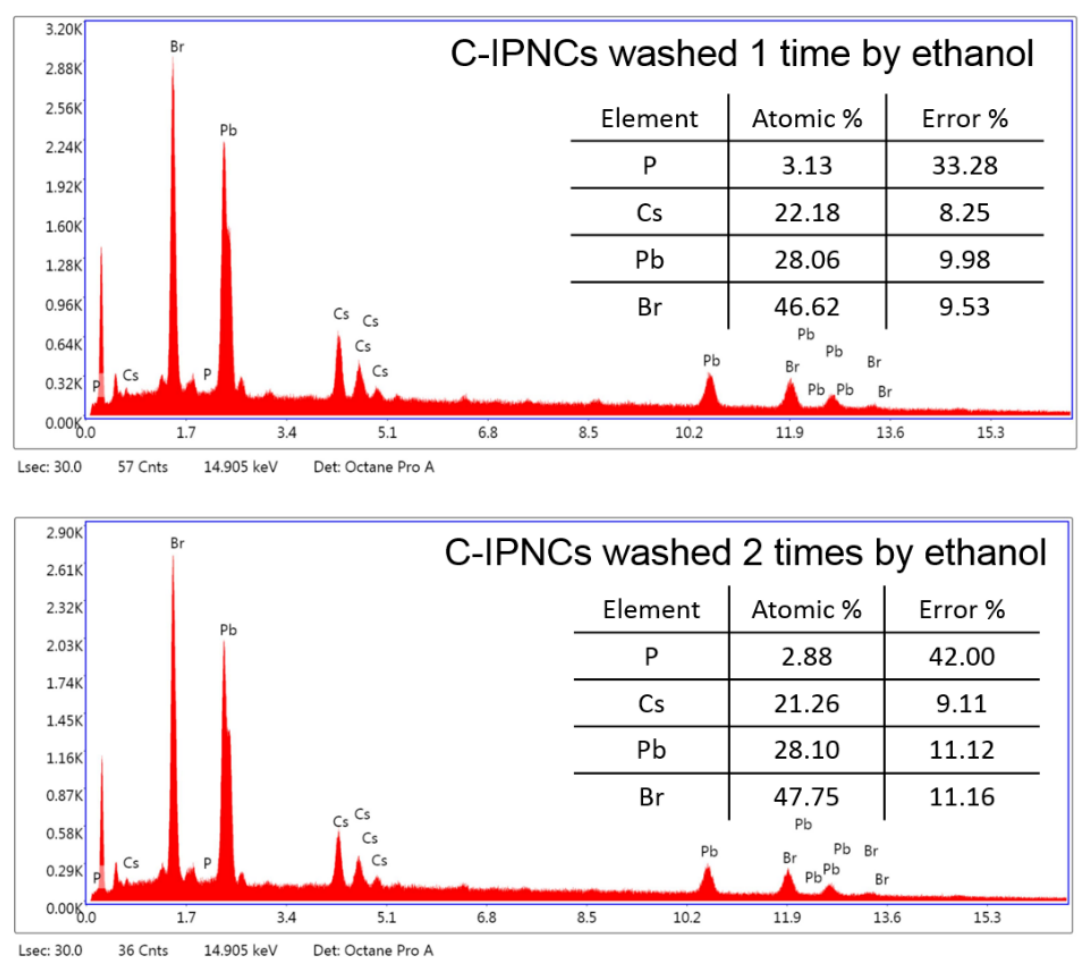

Figure S12. The EDS results of the C-IPNCs samples after washing once and twice by ethanol.

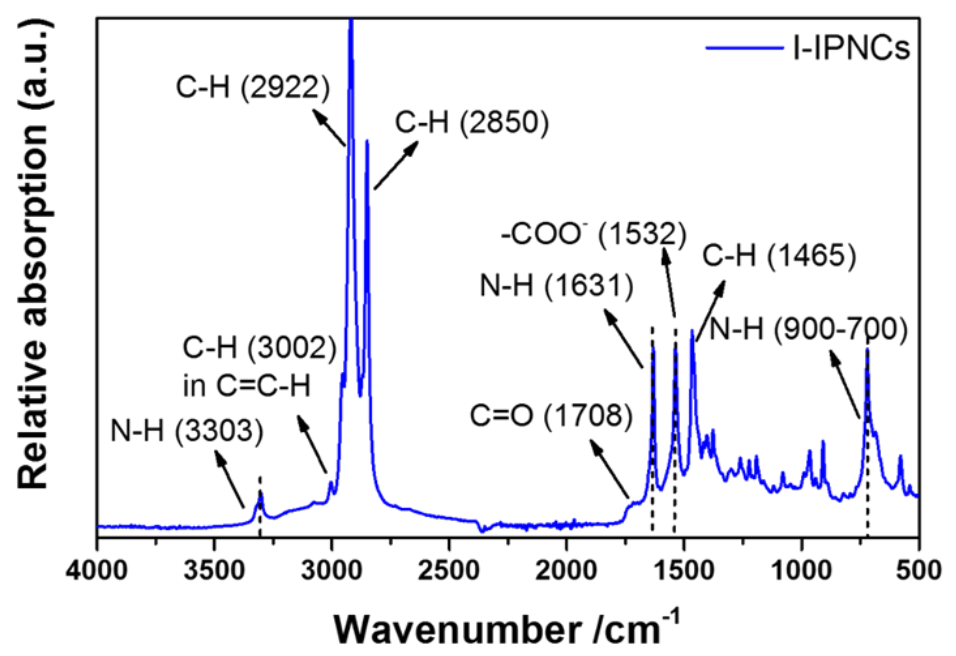

Figure S13. FTIR spectrum of I-IPNCs. The peaks at $3303 \mathrm{~cm}^{-1}, 1631 \mathrm{~cm}^{-1}$ and $900-700 \mathrm{~cm}^{-1}$ are assigned to N-H. The peak at $1532 \mathrm{~cm}^{-1}, 1708 \mathrm{~cm}^{-1}$ and $3002 \mathrm{~cm}^{-1}$ belongs to oleate/oleic acid. 


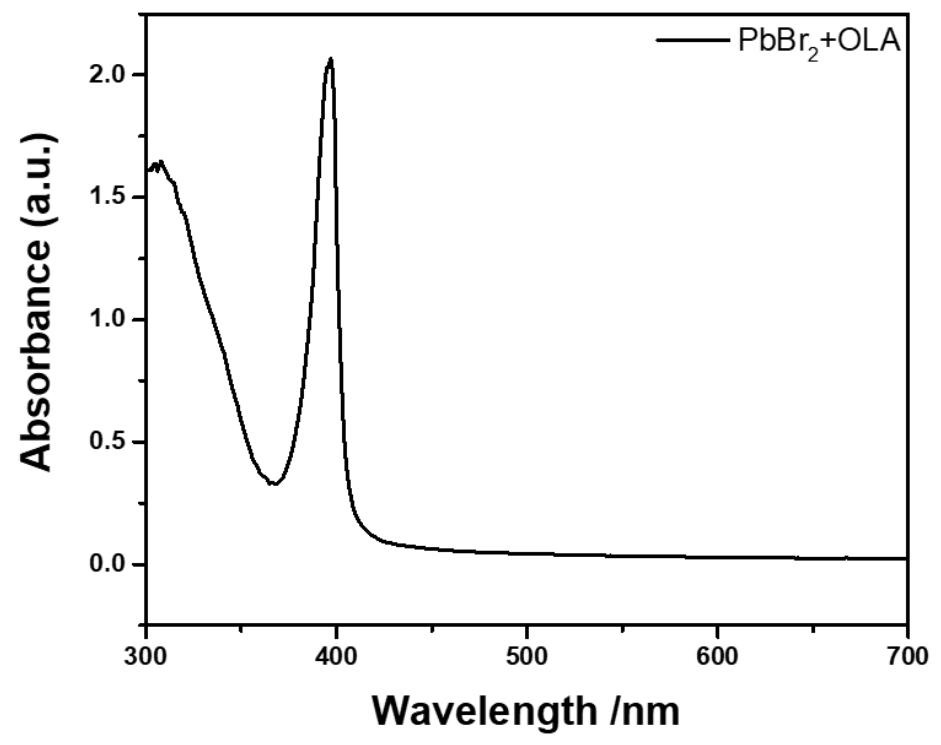

Figure S14. UV-Vis spectra of $\mathrm{PbBr}_{2}$-oleylamine solution in toluene.

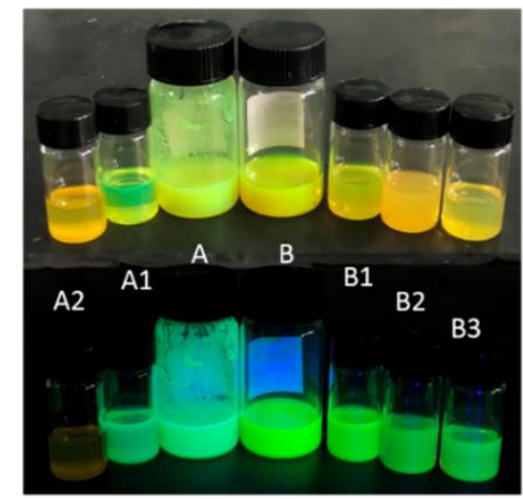

A: Crude I-IPNCs solution

A1: A washed once by ethanol

A2: A washed twice by ethanol

B: Crude C-IPNCs solution

B1: $B$ washed once by ethanol

B2: $B$ washed twice by ethanol

B3: B1 dispersed in ethanol

C1: PL spectra of the C-IPNCS

C2: PL spectra of CdSe QDs
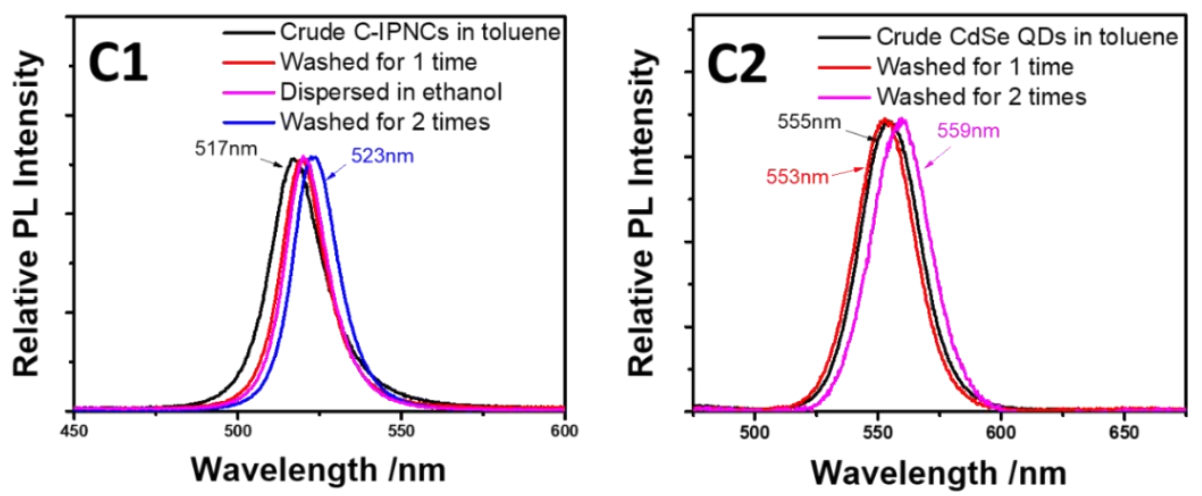

Figure S15. Images of the crude I-IPNCs (A) and C-IPNCs (B) and the samples after washing once and twice by ethanol and dispersed in dimethylbenzene. Normalized PL spectra of the crude C-IPNCs (C1) and CdSe QDs (C2) in dimethylbenzene solution and the samples after washing once and twice, as well as one sample of C-IPNCs after washing once and dispersed in ethanol. 


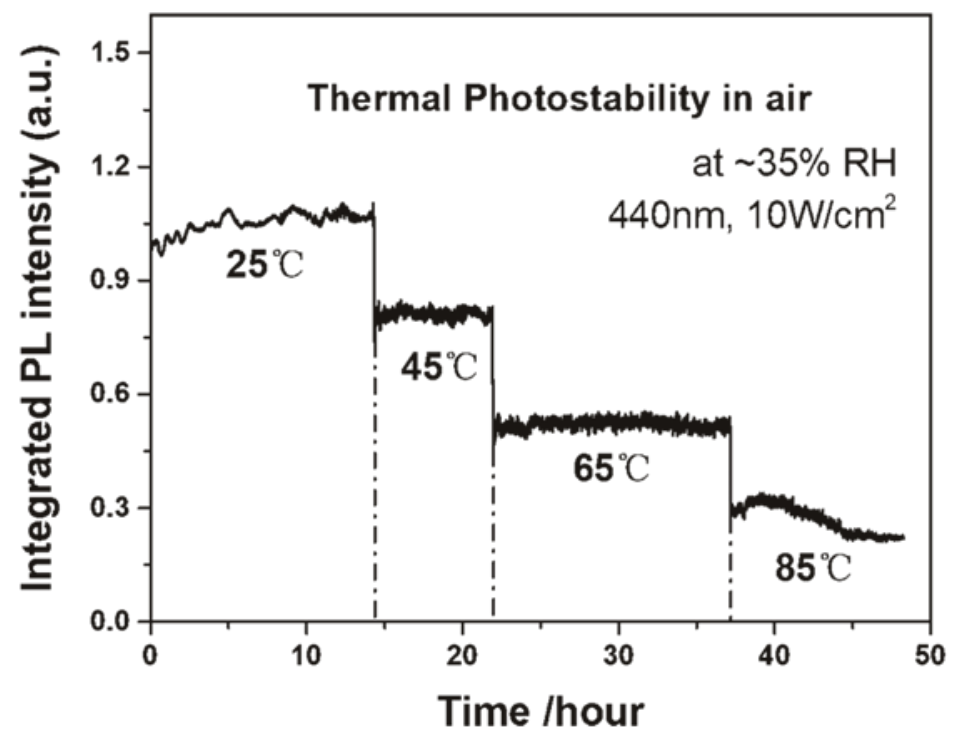

Figure S16. Stability of C-IPNCs at different temperature.

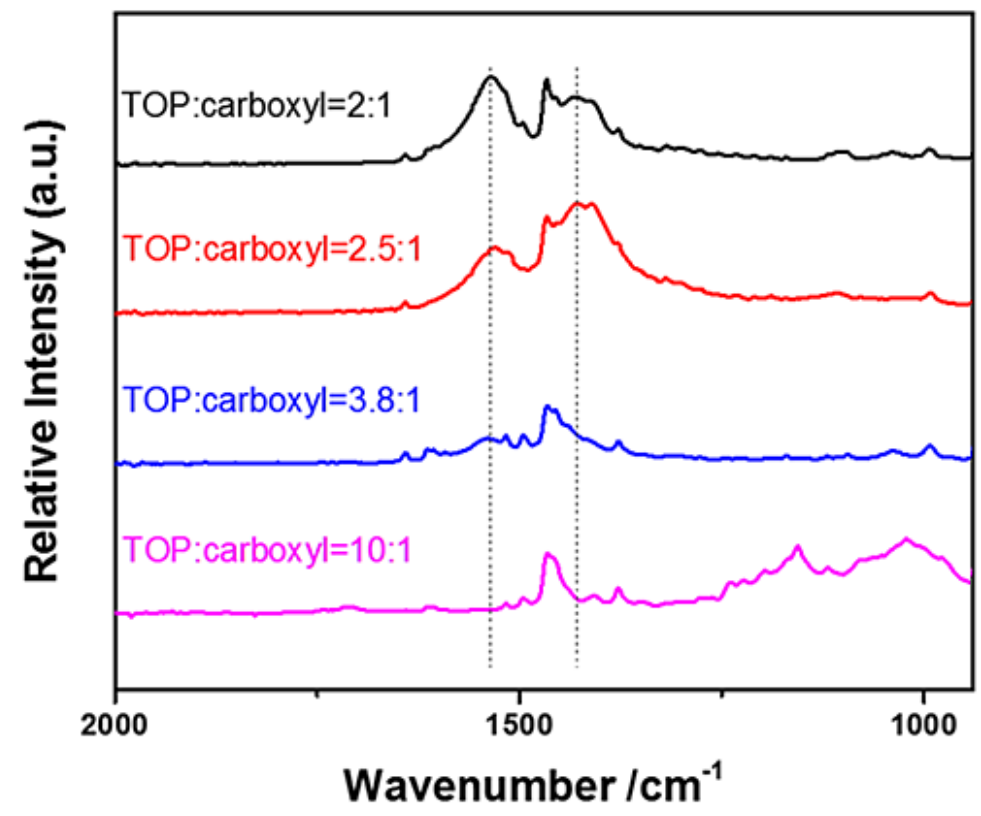

Figure S17. FTIR spectra of C-IPNCs synthesized with different ratios of TOP:carboxyl. 

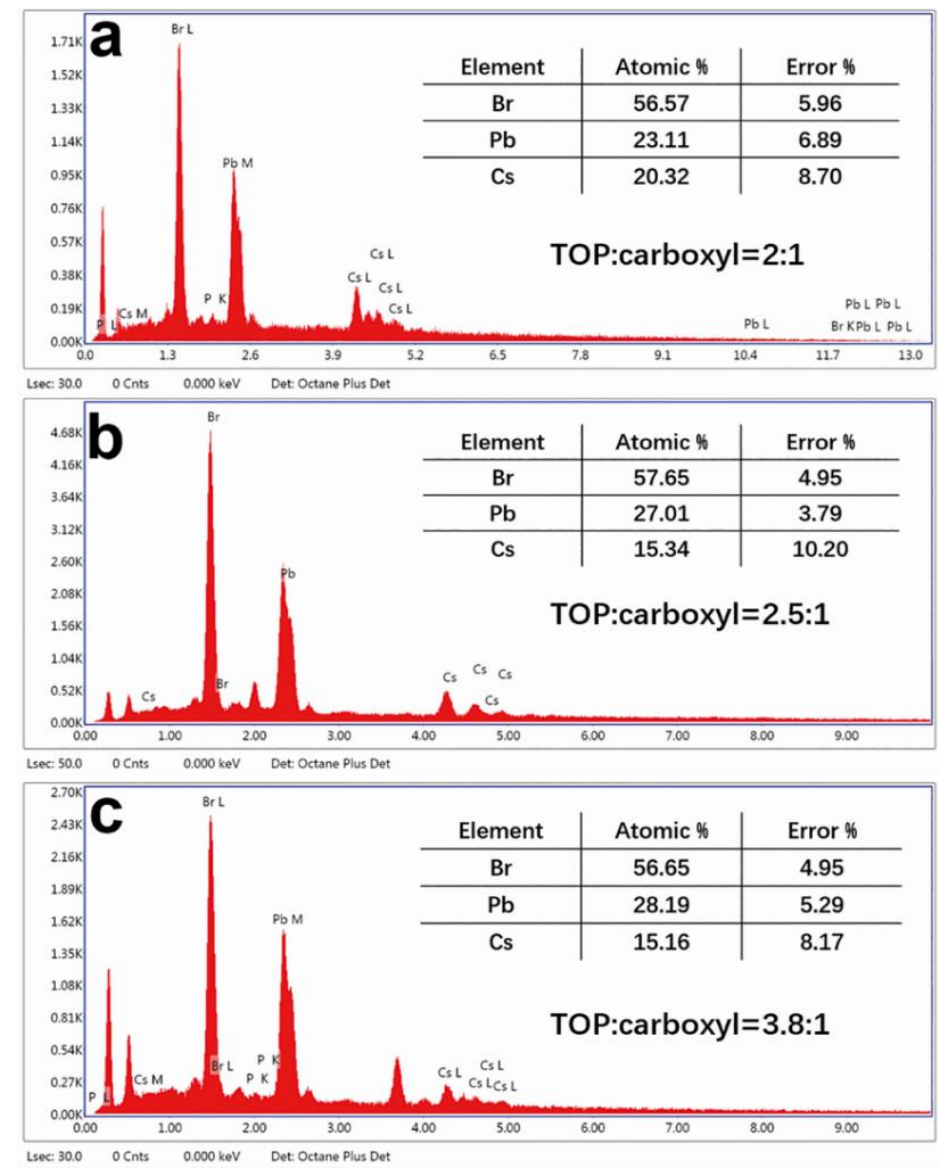

Figure S18. EDS characterization of C-IPNCs prepared with different ratios of TOP:carboxyl.

$$
\begin{aligned}
\mathrm{TOP}+\mathrm{Pb}(\mathrm{St})_{2} & \longrightarrow \mathrm{TOP}-\mathrm{St}+-\mathrm{PbSt} \\
-\mathrm{PbSt}+\mathrm{IPNCs} & \longrightarrow \mathrm{IPNCs}-\mathrm{PbSt} \\
\mathrm{TOP}-\mathrm{St}+\mathrm{Pb}(\mathrm{St})_{2} & \longrightarrow \mathrm{TOP}_{-} \mathrm{St}_{2}+-\mathrm{PbSt} \\
\mathrm{TOP}-\mathrm{St}_{2} & \longrightarrow \mathrm{TOPO}+\mathrm{RC}(=\mathrm{O})-\mathrm{O}-\mathrm{C}(=\mathrm{O})-\mathrm{R}
\end{aligned}
$$

Scheme S1. Proposed reaction scheme between TOP and $\mathrm{Pb}(\mathrm{St})_{2}$ for surface modification of IPNCs.

\section{REFERENCES}

(1) Li, X. M.; Wu, Y.; Zhang, S. L.; Cai, B.; Gu, Y.; Song, J. Z.; Zeng, H. B. Adv. Funct. Mater. 2016, 26, $2435-2445$.

(2) Li, H. B.; Qian, Y.; Xing, X.; Zhu, J. F.; Huang, X. Y.; Jing, Q.; Zhang, W. H.; Zhang, C. F.; Lu, Z. D. J. Phys. Chem. C 2018, 122, 12994-13000. 